\title{
Oral and vocal fold diadochokinesis in dysphonic women
}

\author{
Talita LOUZADA¹, Roberta BERALDINELLE², Giédre BERRETIN-FELIX³ ${ }^{3}$ Alcione Ghedini BRASOLOTTO ${ }^{4}$
}

\author{
1- Speech-Language Pathologist, Bauru School of Dentistry, University of São Paulo, Bauru, SP, Brazil. \\ 2- Speech-Language Pathologist, MSc student, Department of Speech-Language Pathology and Audiology, Bauru School of Dentistry, University of São \\ Paulo, Bauru, SP, Brazil. \\ 3- MSc, PhD, Assistant Professor, Speech Language Pathologist, Department of Speech-Language Pathology and Audiology, Bauru School of Dentistry, \\ University of São Paulo, Bauru, SP, Brazil. \\ 4- MSc, PhD, Assistant Professor, Speech Language Pathologist, Department of Speech-Language Pathology and Audiology, Bauru School of Dentistry, \\ University of São Paulo, Bauru, SP, Brazil.
}

Corresponding address: Profa. Dra. Alcione Ghedini Brasolotto - Faculdade de Odontologia de Bauru, Universidade de São Paulo - Departamento de Fonoaudiologia - Alameda Dr. Octávio Pinheiro Brisolla 9-75, C.P. 73 - 17012-901 - Bauru - SP - Brasil - Phone: +55-14-3235-8332 - e-mail: alcione@usp.br.

Received: July 25, 2010 - Accepted: August 16, 2011

\section{ABSTRACT}

$T^{\text {he }}$ he evaluation of oral and vocal fold diadochokinesis (DDK) in individuals with voice disorders may contribute to the understanding of factors that affect the balanced vocal production. Scientific studies that make use of this assessment tool support the knowledge advance of this area, reflecting the development of more appropriate therapeutic planning. Objective: To compare the results of oral and vocal fold DDK in dysphonic women and in women without vocal disorders. Material and methods: For this study, 28 voice recordings of women from 19 to 54 years old, diagnosed with dysphonia and submitted to a voice assessment from speech pathologist and otorhinolaryngologist, were used. The control group included 30 nondysphonic women evaluated in prior research from normal adults. The analysis parameters like number and duration of emissions, as well as the regularity of the repetition of syllables "pa", "ta", "ka" and the vowels "a" and "i," were provided by the Advanced Motor Speech Profile program (MSP) Model-5141, version-2.5.2 (KayPentax). The DDK sequence "pataka" was analyzed quantitatively through the Sound Forge 7.0 program, as well as manually with the audio-visual help of sound waves. Average values of oral and vocal fold DDK dysphonic and nondysphonic women were compared using the " $t$ Student" test and were considered significant when $p<0.05$. Results: The findings showed no significant differences between populations; however, the coefficient of variation of period (CvP) and jitter of period (JittP) average of the "ka," "a" and "i" emissions, respectively, were higher in dysphonic women (CvP=10.42\%, 12.79\%, 12.05\%; JittP=2.05\%, 6.05\%, $3.63 \%)$ compared to the control group (CVP=8.86\%; $10.95 \%, 11.20 \%$; JittP $=1.82 \%$, $2.98 \%, 3.15 \%)$. Conclusion: Although the results do not indicate any difficulties in oral and laryngeal motor control in the dysphonic group, the largest instability in vocal fold DDK in the experimental group should be considered, and studies of this ability in individuals with communication disorders must be intensified.

Key words: Assessment. Voice. Speech. Dysphonia.

\section{INTRODUCTION}

Knowledge about dysphonia manifestations and the degree of commitment that this change causes in laryngeal and vocal behavior are important for defining rehabilitation direction. In order to do that, procedures are necessary for clinical assessment. Perceptual and acoustic assessments, among other procedures, are commonly performed ${ }^{11,13}$.

Oral and vocal fold diadochokinesis (DDK) is one of the tests that can be applied to perform acoustic analysis. The DDK, or syllable alternating motion rate $^{21}$, is the ability to perform rapidly opposite contractions of relatively simple patterns. The results provide information regarding the patient's neuromotor integration and maturation ${ }^{1}$. Vocal fold 
DDK analysis allows evaluation of neuromuscular integrity of the vocal folds $s^{6,7,17}$ and oral DDK analyzes the ability to perform quick repetitions of speech segments.

The majority of studies referring to DDK involve adult populations with neurologic disorders ${ }^{1,12,14,16,19-21}$. Some studies try to understand the vocal manifestation of dysphonic patients through the perceptual and acoustic analysis of voice. Midi, et al. ${ }^{12}$ (2008) evaluated voice acoustic parameter changes in patients with Parkinson's disease (PD) who were searching for their relationship with motor control. The voices perceptual and acoustic ratings evaluations of $20 \mathrm{PD}$ patients, 12 men and 8 women, were compared with the voices of 20 control subjects of corresponding ages and genders. It was observed that women have poorer voice quality when compared to men. Patients with PD had smaller values for maximum phonation time and DDK compared to the control group, while jitter, shimmer and average fundamental frequency (fO) were similar between the two groups. Although men have poorer performance in DDK than women, these values were not statistically significant. Thus, only a few significant correlations between vocal parameters and motor control were found.

The vocal fold DDK measurement indicates laryngeal gestures of opening and closing of the vocal folds, being that changes in extension and speed movement of vocal folds will reproduce changes in the production rate in patterns of duration and transglottic airflow rate ${ }^{6}$. Therefore, the presence of organic and functional changes in dysphonia cases may relate to DDK results.

The DDK evaluation associated with other clinical assessment procedures is an important resource in understanding the manifestations of individual communication disorders. The assessment of oral and vocal fold diadochokinesis (DDK) in individuals with voice disorders may contribute to the understanding of factors that affect balanced vocal production. Scientific studies that make use of this assessment tool support advances in knowledge in this area and reflect the development of more appropriate therapeutic planning.

Consequently, the objective of this study was to compare the results of oral and vocal fold DDK of dysphonic women to the results of oral and vocal fold DDK in women without vocal disorders.

\section{MATERIAL AND METHODS}

This study has been approved by the Research Ethical Committee of the School of Dentistry of the University of São Paulo, Bauru campus.

The recordings were obtained in an acoustically treated studio, and the patients remained seated with a headset microphone (AKG, model-C444PP) positioned laterally between 60 degrees and $5 \mathrm{~cm}$ from the labial commissure. The emissions were recorded on a Intel ${ }^{\circledR}$ Pentium ${ }^{\circledR} 4$ computer (CPU 2.040 GHz and 256 MB RAM) with a 17" LG Flatron E7015 monitor and a Creative Audigy II sound card. The system also used Sound Forge 7.0 professional audio software (Madison, WI, USA), with a sampling rate of $44.100 \mathrm{~Hz}$ and a 16 bit mono channel.

\section{Patients}

The experimental group was composed of 28 women ranging in age from 19 to 54 years old, diagnosed with dysphonia and referred for a voice assessment by a speech pathologist and otorhinolaryngologist. The control group included 30 nondysphonic women age-matched with the experimental group who were evaluated in previous research with normal adults.

The presence of central neurological diseases and the presence of communication disorders were considered exclusion factors for both groups, with the exception of dysphonia for the experimental group.

\begin{tabular}{|c|c|c|}
\hline Parameters & Unit & Comments \\
\hline Average DDK rate (avR) & /s & $\begin{array}{l}\text { Number of repetition per second, which represents the speed of } \\
\text { DDC }\end{array}$ \\
\hline Average DDK period (avP) & $\mathrm{ms}$ & Average time between vocalizations \\
\hline Standard deviation of DDK period (spP) & $\mathrm{ms}$ & \\
\hline Coefficient variation of DDK period (cvP) & $\%$ & $\begin{array}{c}\text { Measures the degree of rate variation in the period, indicationg the } \\
\text { ability to maintain a constant rate of vocalizations }\end{array}$ \\
\hline Perturbation of DDK period (jitP) & $\%$ & $\begin{array}{l}\text { Measures the degree of variation in cycle-to-cycle period,, } \\
\text { indicationg the ability to maintain a constant rate of vocalizations }\end{array}$ \\
\hline $\begin{array}{l}\text { Coefficient variation of DDK peak intensity } \\
\text { (cvl) }\end{array}$ & $\%$ & $\begin{array}{c}\text { Measures the degree of variation intensity in peak of each } \\
\text { vocalization, indicating the ability to maintain constant intensity of } \\
\text { vocalizations }\end{array}$ \\
\hline
\end{tabular}

Figure 1- Parameters for diadochokinesis (DDK) analyzed by Advanced Motor Speech Profile (MSP) program 


\section{Procedures}

During the oral and vocal fold DDK test isolated emissions were requested; namely, the syllables "pa," "ta" and "ka," the sequence "pataka" and then the vowels "a" and "i." Before the beginning of the test, the patient was requested to repeat the sound as rapidly as possible. The patients practiced the sounds before each recording by repeating as fast as possible, after deep breathing, in a clear and precise way the emissions with a comfortable frequency and intensity in order to understand how to perform the test. Soon thereafter, the subjects were guided to perform the tasks, by receiving a signal with the thumb to begin and to stop the repetition. Each emission recording lasted 8 seconds.

\section{Recording Editing}

The "pa," "ta," "ka," "pataka," "a" and "i" emissions were edited by Sound Forge 7.0 professional audio editing professional. software (Madison, WI, USA). The beginning and end of each sample were excluded, except for four seconds from the third to the sixth second.

\section{Oral and vocal fold DDK analysis}

Monosyllabic emissions "pa," "ta" and "ka" and the vowels " $a$ " and " $\mathrm{i}$ " were filed at sampling rate of $11.025 \mathrm{~Hz}, 16$ bit mono channel and analyzed by Advanced Motor Speech Profile Program (MSP) Model 5141 version 2.5.2 (Kay's Elemetrics Corp., Lincoln Park, NJ, USA), whose parameters are described in Figure 1. The MSP program displays a graphical record of emissions showing the horizontal axis (time in seconds) and the vertical axis (energy in $\mathrm{dB}$ ). To do the DDK count, the program drew a line in the central point at the energy scale of the vertical axis in $d B$. The line of analysis was manually positioned in the corresponding value to the intensity average of the DDK sample, provided by the MSP program, and moved up or down, so the program could count all emissions when necessary (Figure 2).

The DDK of the "pataka" sequence was analyzed quantitatively through the Sound Forge 7.0 program. The count of trisyllables per second was performed manually with the auditory and visual waveform support displayed by the program.

\section{Data analysis}

Average values of oral and vocal fold DDK in dysphonic and nondysphonic women were correlated using the "t Student" test and were considered significant when $p<0.05$.

\section{RESULTS}

Subjects were classified by the types of dysphonia, according to Behlau, et al. ${ }^{2}$ (2001) classification. It was considered primary functional dysphonia when there were no injuries or structural alterations of vocal folds identified by nasolaryngoscopy; secondary functional dysphonia to anatomical inadequacy, in the presence of structural alterations of vocal folds, such as epidermoid cyst, varicosity, stria sulcus; organic functional dysphonia, in the

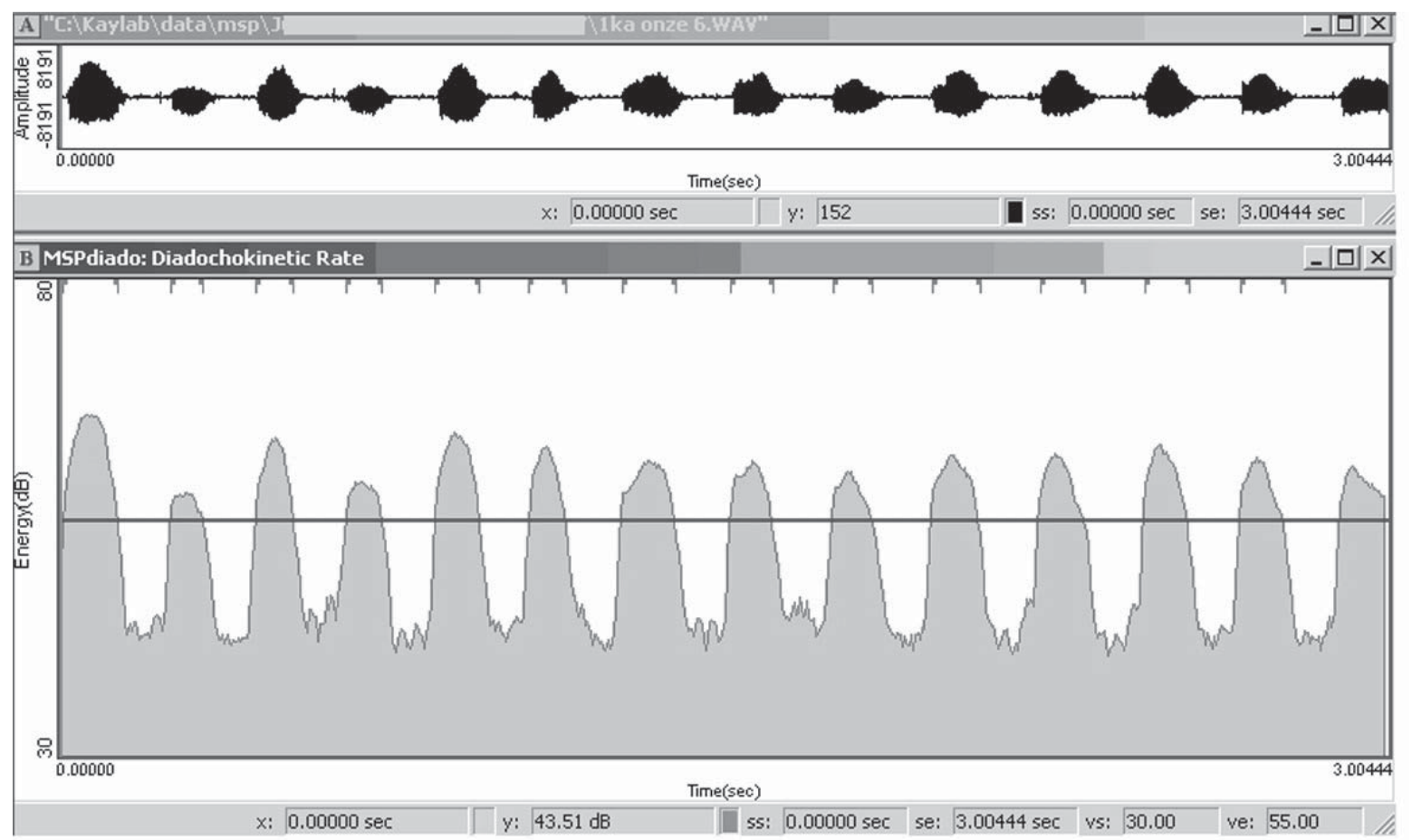

Figure 2-Advanced Motor Speech Profile (MSP) program graphic, where time (seconds) on the horizontal axis and energy $(\mathrm{dB})$ on the vertical axis can be observed 
Table 1- Comparison of oral and vocal fold diadochokinesis (DDK) average and standard deviation, between dysphonic and nondysphonic women, in the emissions of "pa," "ta," "ka," "pataka", "a," and "i."

\begin{tabular}{|c|c|c|c|c|c|c|c|}
\hline Parameters & Group & “pa” & "ta" & “ka” & “a” & “i”" & "pataka" \\
\hline \multirow{5}{*}{ avr (emissions/sec) } & $D$ & 6 & 6.21 & 5.85 & 4.33 & 4.11 & 2.13 \\
\hline & & $( \pm 0.70)$ & $( \pm 0.78)$ & $( \pm 0.73)$ & $( \pm 1.02)$ & $( \pm 1.15)$ & $( \pm 0.34)$ \\
\hline & ND & 5.89 & 5.99 & 5.51 & 4.05 & 3.94 & 2.29 \\
\hline & & $( \pm 0.72)$ & $( \pm 0.85)$ & $( \pm 0.83)$ & $( \pm 1.09)$ & $( \pm 0.88)$ & $( \pm 0.35)$ \\
\hline & $p$ & 0.56 & 0.313 & 0.10 & 0.325 & 0.788 & 0.08 \\
\hline \multirow{5}{*}{ avp (ms) } & $\mathrm{D}$ & 168.86 & 163.60 & 173.69 & 242.5 & 261.79 & - \\
\hline & & $( \pm 20.75)$ & $( \pm 20.76)$ & $( \pm 22.80)$ & $( \pm 52.28)$ & $( \pm 73.88)$ & \\
\hline & ND & 172.27 & 170.98 & 186.8 & 266.13 & 266.62 & - \\
\hline & & $( \pm 23.04)$ & $( \pm 30.53)$ & $( \pm 37.56)$ & $( \pm 78.46)$ & $( \pm 62.30)$ & \\
\hline & $p$ & 0.558 & 0.29 & 0.117 & 0.186 & 0.788 & - \\
\hline \multirow{5}{*}{$\operatorname{sdp}(\mathrm{ms})$} & $D$ & 12.09 & 12.85 & 17.95 & 30.95 & 30.32 & - \\
\hline & & $( \pm 5.68)$ & $( \pm 5.27)$ & $( \pm 11.68)$ & $( \pm 21.85)$ & $( \pm 18.11)$ & \\
\hline & ND & 12.58 & 13.85 & 16.91 & 28.23 & 28.87 & - \\
\hline & & $( \pm 6.14)$ & $( \pm 10.46)$ & $( \pm 11.21)$ & $( \pm 25.47)$ & $( \pm 21.80)$ & \\
\hline & $p$ & 0.756 & 0.651 & 0.73 & 0.665 & 0.785 & - \\
\hline \multirow{5}{*}{ cvp (\%) } & $D$ & 7.14 & 7.87 & 10.42 & 12.79 & 12.05 & - \\
\hline & & $( \pm 3.03)$ & $( \pm 3.10)$ & $( \pm 7.25)$ & $( \pm 9.41)$ & $( \pm 7.07)$ & \\
\hline & ND & 7.29 & 8.01 & 8.86 & 10.95 & 11.2 & - \\
\hline & & $( \pm 3.51)$ & $( \pm 5.54)$ & $( \pm 5.01)$ & $( \pm 8.12)$ & $( \pm 8.73)$ & \\
\hline & $p$ & 0.863 & 0.903 & 0.342 & 0.427 & 0.689 & - \\
\hline \multirow{5}{*}{ jit (\%) } & $D$ & 1.53 & 1.62 & 2.05 & 6.05 & 3.63 & - \\
\hline & & $( \pm 1.03)$ & $( \pm 0.89)$ & $( \pm 1.61)$ & $( \pm 10.65)$ & $( \pm 2.04)$ & \\
\hline & ND & 1.55 & 1.62 & 1.82 & 2.98 & 3.15 & - \\
\hline & & $( \pm 0.96)$ & $( \pm 1.38)$ & $( \pm 0.88)$ & $( \pm 1.46)$ & $( \pm 2.67)$ & \\
\hline & $p$ & 0.92 & 0.992 & 0.504 & 0.123 & 0.453 & - \\
\hline \multirow{5}{*}{ cvi (\%) } & $D$ & 2.36 & 1.92 & 2.55 & 2.49 & 2.26 & - \\
\hline & & $( \pm 1.12)$ & $( \pm 0.99)$ & $( \pm 1.27)$ & $( \pm 1.12)$ & $( \pm 1.16)$ & \\
\hline & ND & 1.85 & 1.76 & 2.12 & 2.2 & 2.57 & - \\
\hline & & $( \pm 0.91)$ & $( \pm 0.86)$ & $( \pm 0.89)$ & $( \pm 1.00)$ & $( \pm 1.24)$ & \\
\hline & $p$ & 0.061 & 0.496 & 0.143 & 0.299 & 0.331 & - \\
\hline
\end{tabular}

$D=$ dysphonic women; $N D=$ nondysphonic women; $p=\%$ of variability

avr= Average DDK rate, avp= Average DDK period, $s d p=$ Standard deviation of DDK period, cvp= Coefficient variation of DDK period, jit= Perturbation of DDK period, cvi= Coefficient variation of DDK peak intensity.

presence of edema, leucoplast or bilateral nodules; and organic dysphonia in cases of gastroesophageal reflux disease or unilateral vocal fold paralysis. Thus, the sample was composed of a participant with primary functional dysphonia, thirteen with secondary functional dysphonia by anatomical inadequacy, seven with organic and functional dysphonia, and three with organic dysphonia, because three women in the sample had no otorhinolaryngologic diagnosis. 
The results concerning the oral and vocal fold DDK of the two groups were compared using the " $t$ Student" test and are described in Table 1.

\section{DISCUSSION}

Although the findings did not show a statistically significant correlation of oral and vocal fold DDK between the group of women with and without voice disorders (Table 1), some aspects about the performance of diadochokinetic tasks are relevant and should be discussed.

The results showed that the DDK speed, the average time between the vocalizations, the ability to keep constant vocalizations and keeping the intensity of these vocalizations constant did not correlate with the presence of dysphonia (Table 1). It was expected that no neurological changes related to vocal production would interfere with oral and vocal fold DDK, since for both tasks there was participation of the opening and closing of vocal folds, especially related to " $a$ " and " $i$ " emissions, that involve specifically alternating motions of glottic adduction and abduction, since organic and functional factors may also interfere with the results of the DDK, including not necessarily expressing a disturbance in oral communication3.

It was observed that in the "pa" emission, the parameter CVI $(p=0.061)$, although not statistically significant, showed a tendency for nondysphonic women to keep the vocalizations more constant as compared to the dysphonic group. This can be explained by the fact that the glottic changes found in dysphonic women, make intensity control difficult, since vocal balance depends on correct coordination between the myoelastic and aerodynamic forces of the laryn $x^{5}$; i.e., less intensity variation. This finding is possibly related to the size of the sample, thus, statistically significant values can be found if the number of subjects were more expressive.

Oral diadochokinesis may be indicative of structural and physiological changes ${ }^{15}$. The impairment of phonoarticulatory organs can generate vocal consequences, such as changes in vocal quality, impaired laryngeal mobility, and extrinsic muscle tension of the laryn ${ }^{8}$. The interference of articulation with phonation shows that such factors might affect the motor performance of these structures and thus, in DDK. Dysphonia may happen as a secondary sign of muscular tension syndrome ${ }^{8}$. Thus, there is a physiological and anatomical inter-connecting of the structures common to phonation and articulation'. Studies refer to DDK as a diagnostic tool for myofunctional changes ${ }^{15,16}$. Thus, individuals who have dysphonia due to a myofunctional alteration that initiates larynx overload could have poorer DDK performance.
Some minor structural alterations have morphological characteristics that change the vibration pattern of vocal folds. Therefore, the sulcus vocalis shows an arched vocal fold ${ }^{4}$, and cysts appear on a bulged vocal fold ${ }^{10}$. This could interfere with the speed of the vocal folds alternating motion rate (DDK). Furthermore, it is minor structural alterations frequently combine with incomplete glottic closure. Leeper, Heeneman and Reynolds ${ }^{6}$ (1990) reported that, when DDK involves vowel emission, refined control of vocal fold opening and closing is required.

Factors such as rigidity and mass can interfere with the fundamental frequency of voice ${ }^{18}$, beyond subglottic pressure, which reflects on vocal intensity. There are significant differences in the effect of frequency and vocal intensity upon the airflow through the vocal folds ${ }^{7}$. Furthermore, vocal intensity seems to be the primary factor controlling the speed of the DDK and airflow during the production of the vowel "a"7.

In this study, although no statistically significant correlation was observed, an average frequency of disturbances in the DDK "a" (DDCjiit) were higher in dysphonic subjects than in the control group, and this value was approximately doubled. High frequency values of DDK disturbance may indicate a lower ability to control the vocal folds' motion during phonation. Vocal fold DDK has not been investigated in detail like an analysis of laryngeal subsystem, as has already occurred with oral DDK ${ }^{17}$.

Nondysphonic individuals have appropriate adjustments in their laryngeal mechanism that allows for quick glottal opening and closing during the production of the consonants and vowels of syllables ${ }^{6}$. This suggests that these adjustments in laryngeal diseases are inadequate, resulting in an increased effort to compensate. Therefore, information obtained with the DDK can be reference for the interpretation of complex tasks ${ }^{14}$.

There is a need for more studies that compare DDK in a control group and in dysphonic individuals, the latter split into distinct groups according to the vocal alteration, thus making it possible to compare diadochokinetic task performance differences in different dysphonias.

\section{CONCLUSION}

Although the results don't indicate any difficulties in oral and laryngeal motor control in the dysphonic group, the major instability in vocal fold DDK in the experimental group should be considered, and studies of this ability in individuals with communication disorders must be intensified. 


\section{ACKNOWLEDGMENTS}

The authors wish to thank the University of São Paulo for granting scholarship for Scientific Initiation to the first author (09.1.1916.25.4 number), which allowed the release of this work as partial results of the study.

\section{REFERENCES}

1- Baken RJ, Orlikoff RF. Speech movements: diadochokinesis. In: Clinical measurement of speech and voice. $2^{\text {nd }}$ ed; San Diego: Singular; 2000. p. 511-74.

2- Behlau M, Madazio G, Feijó D, Pontes P. Avaliação de voz. In: Behlau, M (org.) Voz: o livro do especialista. Rio de Janeiro: Revinter; 2001. p. 105-15.

3- Dworkin JP, Aronson AE. Tongue strength and alternate motion rates in normal and dysarthric subjects. J Commun Disord. $1986 ; 19(2): 115-32$.

4- Hernando M, Cobeta I, Lara A, Garcia F, Gamboa FJ. Vocal pathologies of difficult diagnosis. J Voice. 2008;22(5):607-10.

5- Jiang J, Lin E, Hanson DG. Vocal fold physiology. Otolaryngol Clin North Am. 2000;33(4):699-718.

6- Leeper HA, Heeneman H, Reynolds C. Vocal function following vertical hemilaryngectomy: a preliminary investigation. ] Otolaryngol. 1990;19(1):62-7.

7- Leeper HA, Jones E. Frequency and intensity effects upon temporal and aerodynamic aspects of vocal fold diadochokinesis. Percept Mot Skills. 1991;73:880-2.

8- Lierde KMV, De Bodt M, Dhaeseleer E, Wuyts F, Claeys S. The treatment of muscle tension dysphonia: a comparison of two treatment techniques by means of an objective multiparameter approach. J Voice. 2010;24(3):294-301.
9- Linville SE, Rens J. Vocal tract resonance analysis of aging voice using long-term average spectra. J Voice. 2001;15(3):323-30. 10- Martins RHG, Santana MF, Tavares ELM. Vocal cysts: clinical, endoscopic, and surgical aspects. J Voice. 2011;25(1):107-10. 11- Mehta DD, Hillman RE. Voice assessment: updates on perceptual, acoustic, aerodynamic, and endoscopic imaging methods. Curr Opin Otolaryngol Head Neck Surg. 2008;6(3):2115

12- Midi I, Dogan M, Koseoglu M, Can G, Sehitolgu MA, Gunal DI. Voice abnormalities and their relation with motor dysfunction in Parkinson's disease. Acta Neurol Scand. 2008;117(1):26-34. 13- Nemr K, Amar A, Abrahão M, Leite GCA, Köhle J, Santos AO, et al. Comparative analysis of perceptual evaluation, acoustic analysis and indirect laryngoscopes for vocal evaluation in population with vocal complaint. Braz J Otorhino. 2005;71(1):13-7.

14- Padovani M, Gielow I, Behlau M. Phonarticulatory diadochokinesis in young and elderly individuals. Arq Neuropsiquiatr. $2009 ; 67(1): 58-61$.

15- Prathanee B. Oral diadochokinetic rates in adults. J Med Assoc Thai. 1998;81(10):784-7.

16- Ray J. Orofacial myofunctional therapy in dysarthria: a study on speech intelligibility. Int J Orofacial Myology. 2002;28:39-48. 17- Renout, KA, Leeper HA, Bandur DL. Vocal fold diadochokinetic function of individuals with amyotrophic lateral sclerosis. Am J Speech Lang Pathol. 1995;4:73-80.

18- Roy N, Hendarto $\mathrm{H}$. Revisiting the pitch controversy: changes in speaking (SFF) after management of functional dysphonia. J Voice. 2005;19(4):582-91.

19- Tjaden K, Watlin E. Characteristics of diadochokinesis in multiple sclerosis and Parkinson's disease. Folia Phoniatr Logop. 2003;55:241-59.

20- Wang YT, Kent RD, Duffy JR, Thomas JE. Analysis of diadochokinesis in ataxic dysarthria using the motor speech profile program. Folia Phoniatr Logop. 2009;61(1):1-11.

21- Ziegler W. Task-related factors in oral motor control: speech and oral diadochokinesis in dysarthria and apraxia of speech. Brain Lang. 2002;80(3):556-75. 